\title{
Multi-group covariance matrices for the resolved resonace range of the hafnium isotopes
}

\author{
G. Noguere ${ }^{1, a}$, C. De Saint Jean ${ }^{1}$, P. Siegler ${ }^{2}$, and P. Schillebeeckx ${ }^{2}$ \\ 1 CEA/DEN Cadarache, 13108 Saint-Paul-lez-Durance, France \\ 2 JRC-EC IRMM, Retiseweg, 2440 Geel, Belgium
}

\begin{abstract}
The accuracy of the neutron properties of ${ }^{174} \mathrm{Hf},{ }^{176} \mathrm{Hf},{ }^{177} \mathrm{Hf},{ }^{178} \mathrm{Hf},{ }^{179} \mathrm{Hf}$ and ${ }^{180} \mathrm{Hf}$ have been investigated on the basis of experimental work carried out at the GELINA facility of the Institute for Reference Materials and Measurements. Six transmission data were interpreted with the REFIT code in term of resolved parameters. The propagation of the nuclear data uncertainties up to multi-group cross sections were performed with Monte Carlo techniques in association with conditional probabilities.
\end{abstract}

\section{Introduction}

Hafnium is a ductile metal which does not exist as a free element in nature. The hafnium isotopes ${ }^{174} \mathrm{Hf},{ }^{176} \mathrm{Hf},{ }^{177} \mathrm{Hf}$, ${ }^{178} \mathrm{Hf},{ }^{179} \mathrm{Hf}$ and ${ }^{180} \mathrm{Hf}$ are found combined in zirconium compounds with a respective natural abundance of $0.16 \%$, $5.26 \%, 18.60 \%, 27.28 \%, 13.62 \%$ and $35.08 \%$. Owing to its mechanical and neutronic properties, it has been selected for a long time in reactor engineering to be used as neutron absorbing material holding in steel clad control rods to regulate the fission process.

Some microscopic and integral data have shown the deficiencies of the existing data libraries to accurately describe the nuclear properties of the natural hafnium $[1,2]$. In the epithermal energy range, the underestimation of the reactivity worth measured in the AZUR-Caramel experiment was interpreted as an overestimation of few percents $(\sim 4 \%)$ of the natural hafnium capture cross section [3]. Few years latter, this integral trend was partially confirmed by the Hf capture resonance integral deduced from time-of-flight data measured at the linac facility of the Renssealer Polytechnic Institute (RPI) [4]. The reported value of $1959 \pm 2$ barns is respectively $1.5 \%, 1.7 \%$ and $0.7 \%$ lower than the JEF-2.2 (1989 barns), JEFF-3.0 (1993 barns) and ENDF/B-VI.8 (1972 barns) recommended values. The resonance parameters extracted by Trbovitch were incorporated in the latest version of the European library JEFF-3.1. Unfortunately, this set of resonance parameters is not able to solve the larger part of the observed underestimation of the reactivity worth.

In order to confirm the RPI results, new measurements of the capture and total cross sections were requested [5]. The experimental campaign is in progress at the IRMM in the frame of the NUDAME program. This work is performed in collaboration with Serco Assurance. T. Ware started his Ph.D. thesis in 2006 in order to take an active part in this project.

A parallel evaluation work is in progress at the CEA of Cadarache to obtain relevant isotopic variances and covariances for hafnium. For the thermal energy range, the analysis is based on the data recommended by Mughabaghab [6],

\footnotetext{
${ }^{a}$ Presenting author, e-mail: gilles.noguere@cea.fr
}

the resolved resonance range is based on the transmission data reported in ref. [2] and the modelling of the unresolved resonance range uses the optical model parameters established by V. Avrigeanu [7]. This paper presents preliminary results and gives the isotopic Xmas (172 fine group structure) covariance matrices obtained with the CADTOOL interface [8] in association with the Monte Carlo tool MCFIT [9].

\section{Resonance shape analysis}

The resonance parameters available in the latest version of the European library JEFF-3.1 (below $200 \mathrm{eV}$ ) are those determined by Trbovitch with SAMMY [10]. He analyzed several capture and transmission data measured at RPI. The results reported in ref. [4] take into account the accuracy of the resolution function and the uncertainties coming from the fitting procedure. The final uncertainties are underestimated because the resolution function is not the main experimental contribution that affect the accuracy of the low energy resonance parameters. The contribution of the Doppler broadening has to be included in the adjustment to get relevant information on the global uncertainties. A new analysis of the resolved resonance range is needed to take into account all the experimental sources of uncertainties.

The present work uses six transmission spectra measured at the Institute for Reference Materials and Measurements (IRMM, Geel, Belgique). The measurements were performed with different natural hafnium samples (table 1) at the Doppler station of the GELINA facility. The neutrons are detected by a Li-glass detector located at $26.45 \pm 0.02 \mathrm{~m}$ from the neutron source. The detailed description of the experimental set-up and

Table 1. Thickness of the three hafnium samples at different temperatures [2].

\begin{tabular}{ccc}
\hline $\begin{array}{c}\text { Thickness } \\
(\mathrm{mm})\end{array}$ & $\begin{array}{c}300 \mathrm{~K} \\
\text { atom/barn }\end{array}$ & $\begin{array}{c}77 \mathrm{~K} \\
\text { atom/barn }\end{array}$ \\
\hline 1 & $0.00475 \pm 0.00005$ & $0.00476 \pm 0.00005$ \\
2 & $0.00938 \pm 0.00006$ & $0.00941 \pm 0.00006$ \\
15 & $0.06645 \pm 0.00009$ & $0.06664 \pm 0.00009$ \\
\hline
\end{tabular}



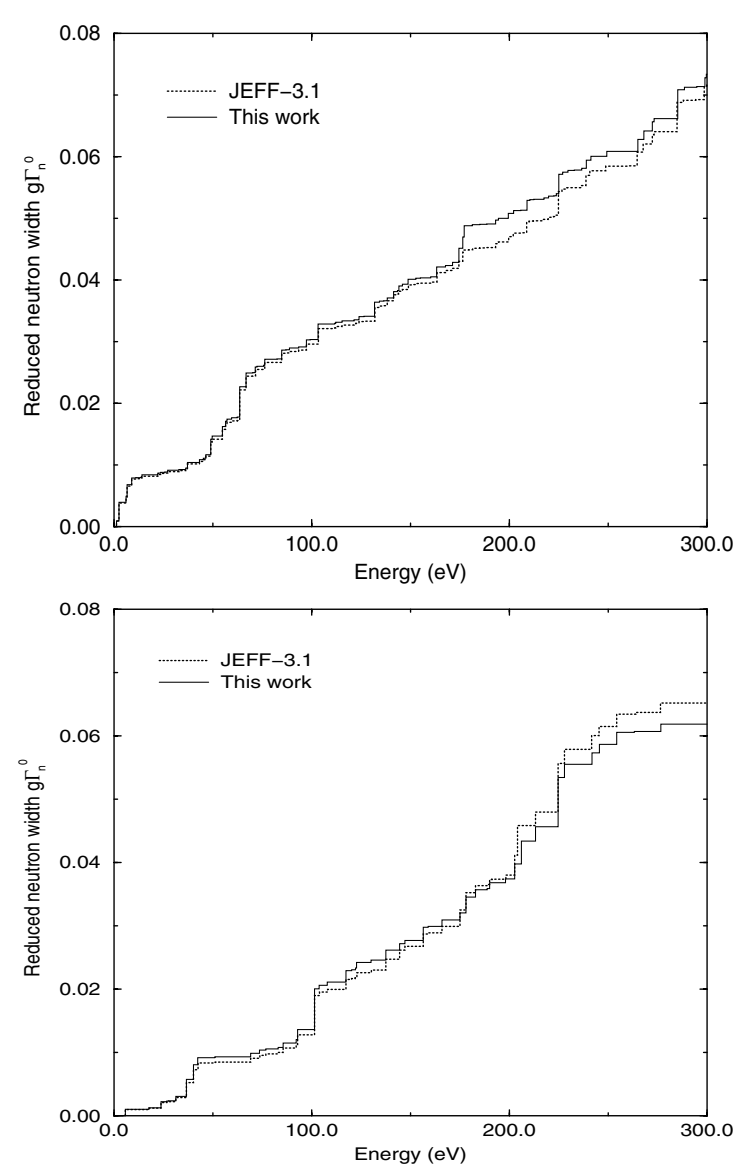

Fig. 1. ${ }^{177} \mathrm{Hf}$ (top plot) and ${ }^{179} \mathrm{Hf}$ (bottom plot) reduced neutron widths obtained in this work up to $300 \mathrm{eV}$ and compared with those recommended in the latest European library JEFF-3.1.

the interpretation of the average transmission spectra with the CALENDF code are given elsewhere [2]. Here, the REFIT code [11] was used to interpret the data in term of ReichMoore parameters up to $300 \mathrm{eV}$. Figure 1 compares the ${ }^{177} \mathrm{Hf}$ and ${ }^{179} \mathrm{Hf}$ reduced neutron widths values obtained in this work with those available in JEFF-3.1. Below $200 \mathrm{eV}$, the results are close to the $g \Gamma_{n}^{0}$ values reported by Trbovich.

\section{Error propagation with the MCFIT tool}

For the resonance analysis, we have used an improved modelisation of the resolution function and determined optimal values for the effective temperatures.

In the REFIT code, the variance and the skewness of the resolution function are respectively determined by two parameters $\lambda_{\mathrm{O}}$ and $\lambda_{\tau}$. They represent the "effective" mean free path of the neutron in the water moderator and in the vicinity of the target-moderator assembly. The value of $\lambda_{0}=$ $5.7 \pm 0.7 \mathrm{~mm}$, determined on ${ }^{56} \mathrm{Fe}$ capture data [12], was confirmed by Monte-Carlo simulations $[13,14]$. To determine $\lambda_{\tau}=55.6 \pm 2.1 \mathrm{~mm}$, we have used the technique proposed in ref. [15], that consists to fit the bottom of the Hf black resonances observed in the transmission of the $15 \mathrm{~mm}$-thick sample.
Table 2. Debye temperature for mono-atomic hafnium crystal.

\begin{tabular}{|c|c|c|c|}
\hline Author & year & ref. & $\theta_{D}(\mathrm{~K})$ \\
\hline Wolcott & 1955 & [17] & 261 \\
\hline Kneip & 1963 & [18] & $\begin{array}{l}252.3 \pm 0.9 \\
251.5 \pm 1.2\end{array}$ \\
\hline Betterton & 1968 & [19] & $252 \pm 1$ \\
\hline Gao & 1999 & [21] & 250 \\
\hline Ostanin ${ }^{\dagger}$ & 2000 & [20] & 215 \\
\hline Feranchuk $^{\dagger}$ & 2002 & [22] & 280 \\
\hline \multicolumn{2}{|c|}{ Average value } & 251.7 & \\
\hline \multicolumn{2}{|c|}{ Standard deviation } & & 19.3 \\
\hline
\end{tabular}

$\dagger$ Calculated value.

The effective temperature introduced in the REFIT analysis of the transmission data measured at $T=300 \pm 1 \mathrm{~K}$ was calculated using a simple Einstein model valid for pure element above room temperature:

$$
T_{e f f} \simeq T_{E} \operatorname{coth}\left(\frac{T_{E}}{T}\right) .
$$

The first two terms of the Taylor expansion of this expression suffice and give [16]:

$$
T_{e f f} \simeq T\left[1+\frac{1}{3}\left(\frac{T_{E}}{T}\right)^{2}\right]
$$

in which $T_{E}$ is the Einstein temperature which may be related to the Debye temperature $T_{E}=\frac{3}{8} \theta_{D}$. Table 2 gives some experimental and calculated Debye temperature for $\mathrm{Hf}$ reported in the literature. Although a large discrepancy is observed between the calculated $\theta_{D}$, the average value remains consistent with the value of $252 \mathrm{~K}$ reported in ref. [23]. By introducing the average value of $\theta_{D}$ and its standard deviation in equation (2), we obtained an effective temperature equal to $T_{\text {eff }}=309.8 \pm 1.8 \mathrm{~K}$. For the transmission data measured at low temperature, $T_{\text {eff }}$ was fitted on the data together with the resonance parameters. The results are shown in figure 2 . The average value is $T_{e f f}=99.4 \mathrm{~K}$ and the standard deviation of the distribution is close to $11.5 \mathrm{~K}$.

In the final analysis, the accuracy of the effective temperature, of the resolution function, of the flight path length and of the sample thickness were included in the fitting procedure by using the Monte Carlo interface MCFIT [9]. This fortran tool propagates the experimental uncertainties and the accuracy of

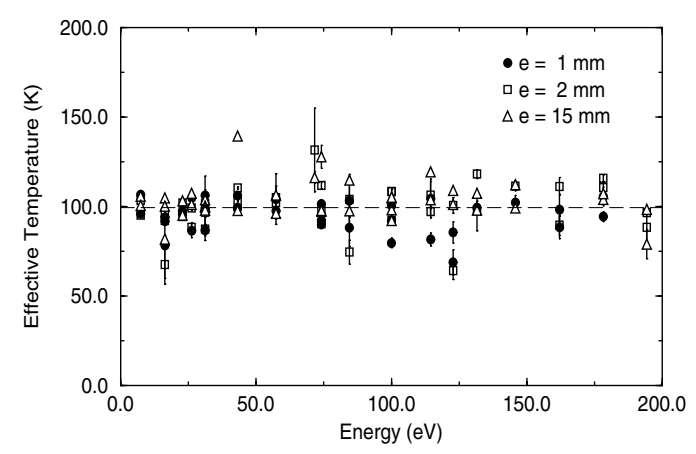

Fig. 2. Effective temperature determined with the REFIT code [11]. 
Table 3. Example of ${ }^{177} \mathrm{Hf}$ resonance parameters obtained in this work. The statistical uncertainty is given by REFIT [11], while the total uncertainty is provided by MCFIT [9].

\begin{tabular}{|c|c|c|c|c|c|}
\hline \multicolumn{2}{|c|}{ Parameter } & JEFF-3.1 & Value & Stat. & Tot. \\
\hline$\overline{E_{n}}$ & $(\mathrm{eV})$ & 2.39 & 2.38 & \pm 0.0003 & \pm 0.009 \\
\hline$\Gamma_{\gamma}$ & $(\mathrm{meV})$ & 60.7 & & & \\
\hline$\Gamma_{n}$ & $(\mathrm{meV})$ & 8.04 & 8.29 & \pm 0.01 & \pm 0.1 \\
\hline$E_{n}$ & $(\mathrm{eV})$ & 5.90 & 5.903 & \pm 0.0001 & \pm 0.009 \\
\hline$\Gamma_{\gamma}$ & $(\mathrm{meV})$ & 62.0 & 63.1 & \pm 0.3 & \pm 1.0 \\
\hline$\Gamma_{n}$ & $(\mathrm{meV})$ & 5.32 & 5.35 & \pm 0.01 & \pm 0.07 \\
\hline$\overline{E_{n}}$ & $(\mathrm{eV})$ & 6.58 & 6.58 & \pm 0.0001 & \pm 0.01 \\
\hline$\Gamma_{\gamma}$ & $(\mathrm{meV})$ & 55.6 & 53.1 & \pm 0.3 & \pm 2.0 \\
\hline$\Gamma_{n}$ & $(\mathrm{meV})$ & 8.21 & 8.62 & \pm 0.03 & \pm 0.2 \\
\hline$E_{n}$ & $(\mathrm{eV})$ & 8.88 & 8.88 & \pm 0.0001 & \pm 0.01 \\
\hline$\Gamma_{\gamma}$ & $(\mathrm{meV})$ & 57.3 & 59.8 & \pm 0.3 & \pm 1.3 \\
\hline$\Gamma_{n}$ & $(\mathrm{meV})$ & 5.89 & 5.84 & \pm 0.01 & \pm 0.08 \\
\hline$\overline{E_{n}}$ & $(\mathrm{eV})$ & 10.96 & 10.96 & \pm 0.0002 & \pm 0.02 \\
\hline$\Gamma_{\gamma}$ & $(\mathrm{meV})$ & 57.0 & 64.9 & \pm 0.8 & \pm 1.9 \\
\hline$\Gamma_{n}$ & $(\mathrm{meV})$ & 0.49 & 0.512 & \pm 0.002 & \pm 0.007 \\
\hline
\end{tabular}

the fixed model parameters. Monte-Carlo techniques are used in association with conditional probabilities to give a realistic covariance matrix associated to the model parameters [24]. Examples of results are given in table 3.

The treatment of the uncertainties implemented in the MCFIT tool is of great importance to get reliable uncertainties on the average parameters. For the capture cross section, the accuracy of the average radiation width plays a crucial role in the determination of the multi-group covariance matrices. The statistical analysis of our ${ }^{177} \mathrm{Hf}$ resonance parameters gives the following result:

$$
\left\langle\Gamma_{\gamma}(A=177)\right\rangle=58.9 \pm 1.9 \mathrm{meV} .
$$

This average value was determined by fitting the total width of 8 resonances below $30 \mathrm{eV}$. This result agrees with the average value of $57 \pm 13 \mathrm{meV}$ reported in ref. [4] and the recommended value of $65 \pm 5 \mathrm{meV}$ given in ref. [6]. The analysis of the unresolved resonance range will allow to verify if the significant reduction of the variance proposed in this work is compatible with the experimental data available in the fast neutron energy range.

\section{Production of multi-group covariance matrices with the CADTOOL interface}

The propagation of the microscopic uncertainties in semiintegral data and multi-group cross sections was performed with the CADTOOL interface [8]. This interface was improved to combine the covariances between the Reich-Moore parameters of the Hf isotopes (obtained with MCFIT below $30 \mathrm{eV}$ ) with independent uncertainties reported in the literature $[4,6]$. The long range correlations were introduced with the average radiation width and its uncertainty.

The ${ }^{177} \mathrm{Hf}$ capture and total cross sections obtained in the thermal energy range are shown in figure 3 and compared with the EXFOR data. The accuracy of our evaluated cross sections
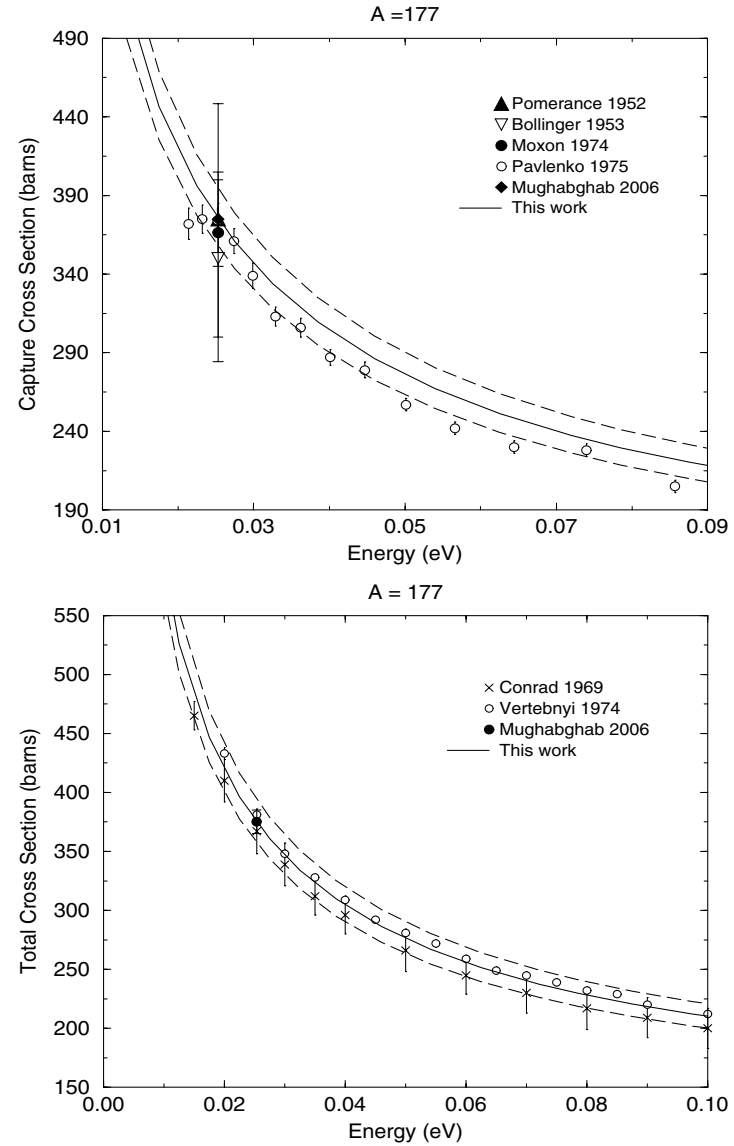

Fig. 3. ${ }^{177} \mathrm{Hf}$ capture and total cross sections obtained in this work and compared with data reported in the literature.

remains within the limit of the experimental uncertainties. The value of the thermal capture cross section is:

$$
\sigma_{\gamma}=374 \pm 18 \text { barns. }
$$

This results is in excellent agreement with the recommended value of $375 \pm 10$ barns reported in ref. [6].

A set of Xmas covariance matrices were obtained by running the GROUPR module of the NJOY code via the CADTOOL interface [8]. The correlations coefficients for the capture cross section are shown in figure 4 . As expected, the contribution of the negative resonances dominates in the low energy range and the values of the corresponding groups are strongly correlated up to few eV, except for ${ }^{177} \mathrm{Hf}$. For this isotope, the thermal capture cross section is entirely defined by the nuclear properties of the internal levels. The contribution of the tails of the bound levels is negligible compared to the strength of the broad s-waves at $1.1 \mathrm{eV}$ and $2.4 \mathrm{eV}$.

\section{Conclusions}

This work demonstrates the possibility to get isotopic multigroup covariance matrices by mixing various correlated or independent sources of uncertainties. One of the remaining question is about the correct propagation of the accuracy of the radiation widths that are set to the average value. 

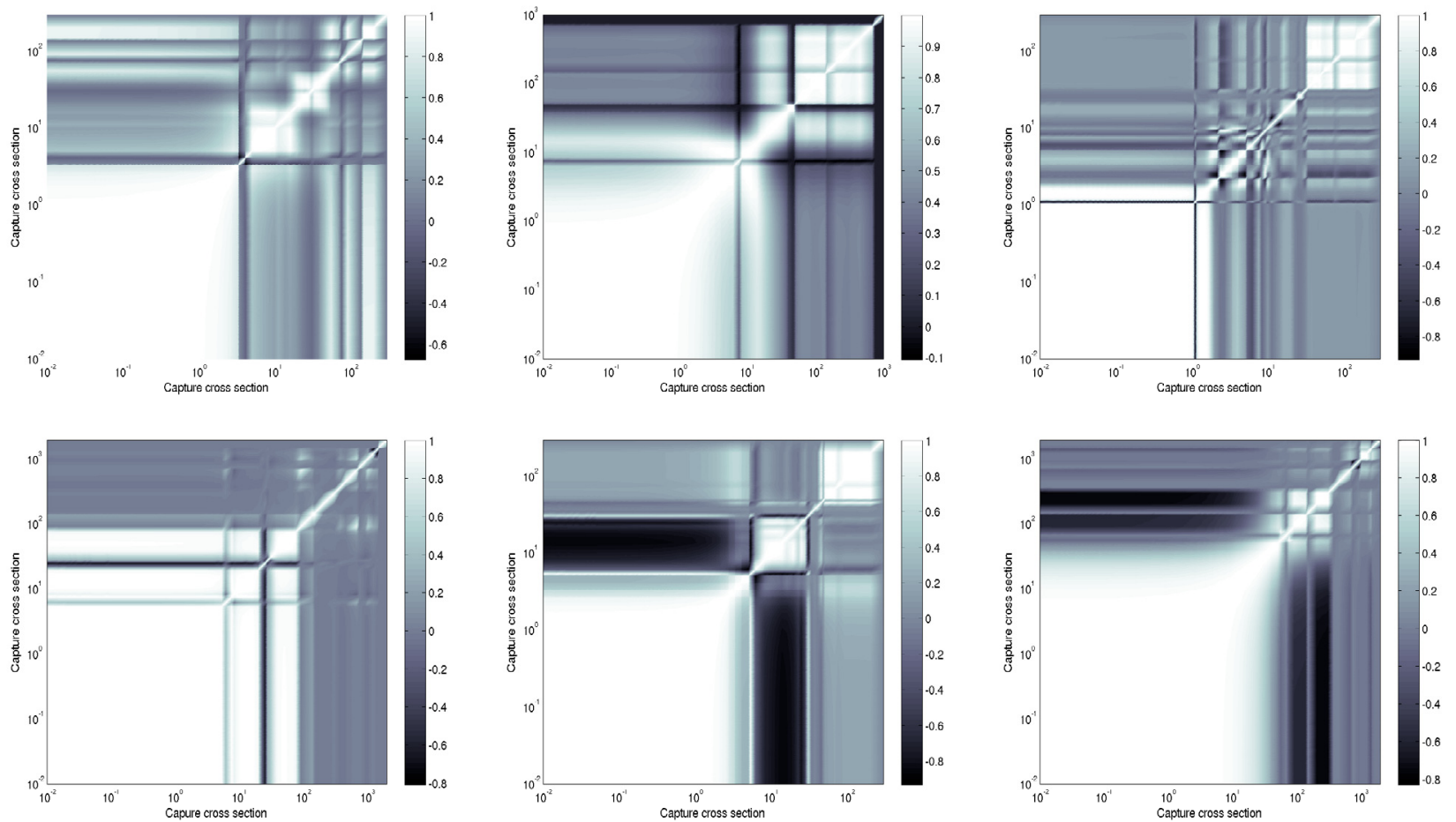

Fig. 4. Isotopic multi-group covariance matrices for the resolved resonance range of the ${ }^{174} \mathrm{Hf},{ }^{176} \mathrm{Hf},{ }^{177} \mathrm{Hf},{ }^{178} \mathrm{Hf},{ }^{179} \mathrm{Hf}$, and ${ }^{180} \mathrm{Hf}$ isotopes obtained with the CADTOOL interface [8].

Here, the average radiation width are sampled according to its mean value and standard deviation. A better solution could be to calculate the average radiation width at each iteration. This approach will be more consistent with the treatment of the unresolved resonance range.

\section{References}

1. K. Dietze et al., Integral test of neutron data and comparison of codes by re-analysis of the SEG and STEK experiments, OECD, NEA JEF/DOC-861 (2001).

2. P. Siegler, K. Dietze, P. Ribon, Nucl. Sci. Tech. Suppl. 2, 936 (2002).

3. J.M. Palau, CEA Cadarache, Report CEA-R-5843, 1999.

4. M. Trbovich, Ph.D. thesis, Faculty of Rensselaer Polytechnic Institute, 2003.

5. A. Plompen et al., The NEA high priority nuclear data request list for future needs (these proceedings).

6. S.F. Mughabghab, Atlas of Neutron Resonances, 5th edn. (Elsevier, Amsterdam, 2006).

7. V. Avrigeanu et al., Nucl. Phys. A 765, 1 (2006).

8. G. Noguere, D. Bernard, C. De Saint-Jean, in Proceedings of the Workshop On Nuclear Data and Evaluation for Reactor applications, Oct. 9-11, Château de Cadarache, Saint-Paul-lezDurance, France, 2006.
9. G. Noguere, C. De Saint Jean, B. Iooss et al., in Proceedings of PHYSOR-2006 Topical Meeting on Reactor Physics, Vancouver, $B C$, Canada, 2006 (to be published).

10. N.M. Larson, Oak Ridge National Laboratory Report ORNL/TM-9179/R7, 2006.

11. M.C. Moxon, J.B. Brisland, Harwell Laboratory Report CBNM/ST/90-131/1, 1990.

12. F. Corvi et al. (private communication).

13. C. Coceva, R. Magnani, IRMM report No. GE/R/ND/96, 1996.

14. M. Flaska et al., Nucl. Instrum. Meth. A 531, 392 (2004).

15. V. Gressier, CEA Saclay Report DAPNIA/SPHN-99-04T, 1999.

16. H.J. Stone et al., J. Appl. Phys. 98, 064905 (2005).

17. N.M. Wolcott, in Proceedings of the Conférence de physique des basses température, Paris, 1955 (Centre National de la Recherche Scientifique and UNESCO, Paris, 1956), p. 286.

18. G.D. Kneip et al., Phys. Rev. 130, 1687 (1963).

19. J.O. Betterton, J.O. Scarbrough, Phys. Rev. 168, 715 (1968).

20. S.A. Ostanin, V.Yu. Trubitsin, Comput. Mater. Sci. 17, 174 (2000).

21. H.X. Gao, L.-M. Oeng, Acta. Cryst. 55, 926 (1999).

22. I.D. Ferenchuk, A.A. Minkevich, A.P. Ulyanenkov, Eur. Phys. J. Appl. Phys. 19, 95 (2002).

23. C. Kittel, Introduction to Solid State Physics, 7th edn. (Wiley, New York, 1995).

24. C. De Saint Jean, G. Noguere, B. Iooss, in Proceedings of PHYSOR-2006 Topical Meeting on Reactor Physics, Vancouver, $B C$, Canada, 2006 (to be published). 